\title{
A Computational Efficient Method For Bandwidth Extension of a Conference Phone
}

\author{
Fredric Lindstrom, Student Member, IEEE, Mattias Dahl, Member, IEEE, Ingvar Claesson, Member, IEEE
}

\begin{abstract}
This paper presents a computationally efficient method for extension of the bandwidth of a conference telephone. The proposed method allows an improvement in quality, i.e. increased bandwidth, at a negligible extra computational cost. This is performed by a combination of an acoustic echo cancellation unit and an adaptive gain unit. The proposed method was implemented in a real-time system. Frequency analysis in combination with subjective tests showed that the proposed method extends the bandwidth with high quality ${ }^{\text {. }}$
\end{abstract}

Index Terms - Hands-Free, Bandwidth Extension, Acoustic Echo Cancellation, Adaptive Gain.

\section{INTRODUCTION}

$\mathrm{E}$ XTENDING the bandwidth of a conference phone can become attractive when e.g. an PSTN phone is equipped with an ISDN interface or connected to a video conference system. This paper presents a method suitable when extending the bandwidth of an existing full-duplex conference phone using Acoustic Echo Cancellation (AEC). The proposed method demands only an adaptive gain technique for the extension. The core of an AEC is a continuously updating adaptive filter [1]. Examples of updating algorithms potential for real-time implementations of an AEC are Normalized Least Mean Square (NLMS) [2], Affine Projection Algorithm (APA) [3], and possibly Fast Transversal Filter (FTF), [4], [5]. Out of these the NLMS algorithm is the most popular algorithm, due to its low complexity and the robustness to finite precision errors. The key parameter in the NLMS algorithm is the step-size, i.e. the pace of the adaptive filter update. State-of-the-art step-size management is found in [6]. Examples of half-duplex systems using only gain techniques can be found in [7] - [10]. In this paper, a combined AEC and adaptive gain structure is proposed. This structure allows a simple extension of the bandwidth of an existing conference phone, without any significant increase in computational complexity. Further, an algorithm for the adaptive gain part of the structure is provided.

\footnotetext{
${ }^{1}$ This work was supported in part by the Swedish Knowledge Foundation (KKS).

F. Lindstrom is with Konftel Technology AB, Research and Development, Box 268, S-90106, Umea, Sweden (e-mail: fredric.lindstrom @konftel.com).

M. Dahl is with Blekinge Institute of Technology, Department of Signal Processing, S-37225, Ronneby, Sweden. (e-mail: mattias.dahl@bth.se)

I. Claesson is with Blekinge Institute of Technology, Department of Signal Processing, S-37225, Ronneby, Sweden. (e-mail: ingvar.claesson @bth.se)
}

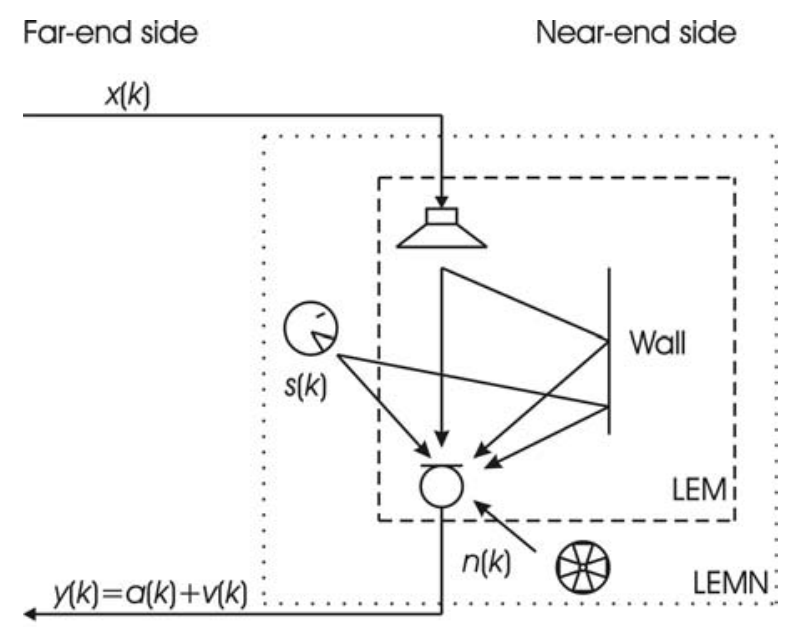

Fig. 1. Signals applying to the AEC, the LEM system (dashed), and the LEMN system (dotted)

\section{The Acoustic ECHO Problem}

The hands-free environment is here described using the following notation, see Fig.1. The far-end signal is denoted $x(k)$, the near-end signal, $y(k)$, the acoustic echo, $a(k)$, and the near-end generated sound, $v(k)$, where $k$ is the sample index. Near-end sound consists of the sum of the near-end talk, $s(k)$, and near-end background noise, $n(k)$, filtered by the room and the microphone, see Fig.1. The near-end talk $s(k)$ consists of a direct path part and a part that reaches the microphone via reflections. The reflected part is referred to as the near-end room reverberation signal. Near-end room reverberation can also degenerate the perceived speech quality. However, the acoustic echo suppression is the focus of this paper. The acoustic echo $a(k)$ is the result of the transformation of the farend signal $x(k)$ as it passes through the loudspeaker, the room and the microphone. The near-end signal is thus a combination of the acoustic echo and the near-end generated sound, i.e. $y(k)=a(k)+v(k)$. The combined influence from the loudspeaker, the room, and the microphone on a signal that passes through all of these is denoted the Loudspeaker Enclosure Microphone (LEM) system. The combined operation of filtering with the LEM system and adding the near-end generated sound is denoted as the LEMN system, see Fig.1. The LEMN system can thus be seen as a transformation of the far-end signal $x(k)$ into the acoustic echo $a(k)$ and addition of the near-end generated sound $v(k)$, in order to produce the near-end signal $y(k)$. The acoustic echo problem can be loosely formulated as: Design a hands-free system where the acoustic echo $a(k)$ in the return line, disturbing the far-end talker, is negligible.

Four different situations of speech activity apply to a 
hands-free telephony. These are; idle, i.e. nobody is talking, near-end talk, i.e. only the near-end talker(s) are active, farend talk, i.e. only the far-end talker(s) are active, and doubletalk, i.e. both sides are talking simultaneously. The doubletalk situation is the most demanding, due to simultaneous transmission in both directions. If a solution to the acoustic echo problem can handle the doubletalk state without interrupting communication in any direction, it is denoted a full-duplex system.

\section{The Adaptive Gain Solution}

An adaptive gain solution to the acoustic echo problem is depicted in figure 2. In this solution the acoustic echo is suppressed by damping the line-out signal and/or the loudspeaker signal. A classical problem for this solution is the intrinsic half-duplex character of the system, i.e. in doubletalk one direction of communication is always damped. However, the effect from the gain factors on the perceived character of the system is a subjective matter, why general statements regarding the quality of an adaptive gain solution should be used with caution.

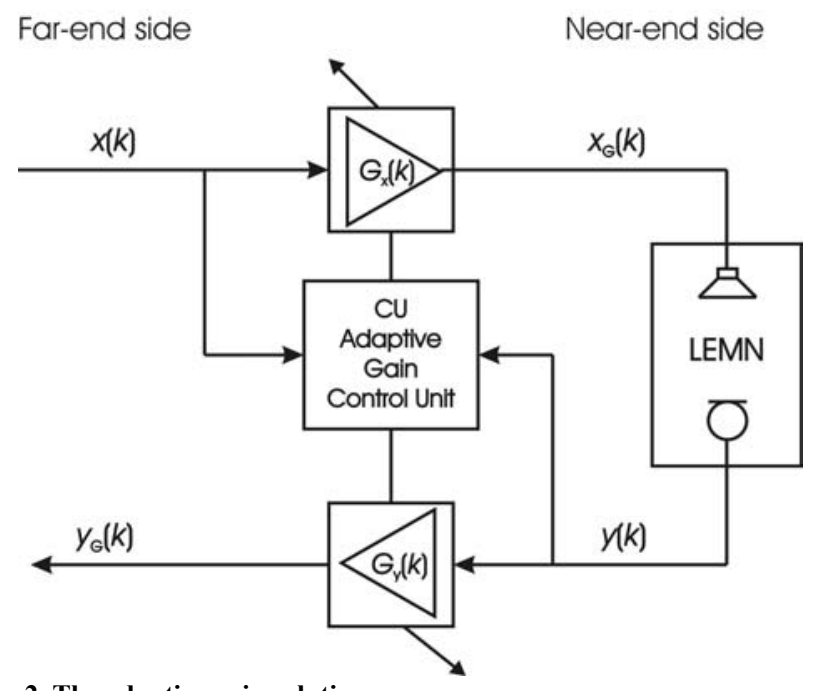

Fig. 2. The adaptive gain solution

\section{The Acoustic Echo Canceling Solution}

An AEC based on a system identification scheme is depicted in Fig.3. It consists of an Adaptive Processing Unit (APU), which in its turn consists of an adaptive filter and an algorithm for the adaptation of the filter. The purpose of such an AEC is to adapt the transfer characteristics of the adaptive filter to be similar to the LEM. Thereby a replica of the acoustic echo, $\hat{a}(k)$, can be produced. The echo cancelled line out signal, $e(k)$, is then obtained by subtracting the replica from the microphone signal, i.e. $e(k)=y(k)-\hat{a}(k)$. In situations of far-end talk the line out signal only contains the estimated error of the replica. In these situations the filter of the APU is adapted using $e(k)$ as an error feed-back signal. There is no damping of either the microphone or the loudspeaker signal in the AEC solution. Real implementations of hands-free systems will most likely contain more or less damping in order to maintain robustness of the system. Still such real AEC based solutions are generally characterized as full-duplex as long as simultaneous two-way communication can be performed with satisfying quality. With this interpretation, the criterion for the full-duplex characterization is strongly subjective.

\section{Far-end side Near-end side}

\section{$x(k)$}

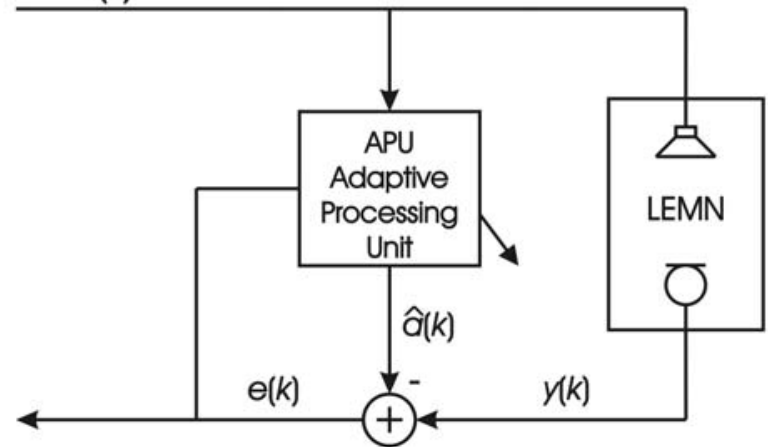

Fig. 3. The acoustic echo canceling solution

\section{THE PROPOSED SOLUTION}

\section{A. Motivation}

The aim of the proposed solution is to provide a mean for the extension of the bandwidth of an existing AEC conference phone. The core of the proposed method is to extend the bandwidth by adding a high frequency band where the acoustic echo is suppressed by an adaptive gain solution. The main motive for using this method is that the bandwidth can be extended without any significant increase in computational complexity, se below. Further, a speech signal is dominated by low frequency content, [11]. The AEC uses speech signals for the adaptation and thus the high frequency part of the LEM estimate is harder to mimic. When the bandwidth of an existing well performing AEC is extended by a direct increase of the sample rate, the AEC might not be able to cancel the high frequency components of the acoustic echo. Finally, in the proposed method, no assumptions on the structure of the AEC at hand are made and no signals from the AEC are used. Thus, the proposed method is well suited to use in conjunction with any existing AEC conference phone.

\section{B. Structure}

The proposed solution implies a structure where both speech signals, (i.e. the far-end and the near-end signals), are split in two frequency bands, one that contains the lower frequencies and one that contains the higher frequencies, which are processed in different ways, see Fig.4. The low frequency part is processed with a conventional full duplex AEC. Acoustic echoes in the low frequency band will therefore be cancelled and communication will not be interrupted in either direction. The high frequency part will be passed with a level dependent 
damping, i.e. high frequency echoes are suppressed with a damping factor. This implies that in a situation of acoustic echo, the high frequencies of the near-end signal will be damped. The method is depicted in Fig.4 where the far-end signal, $x(k)$, is divided into a low frequency part, $x_{\mathrm{L}}(k)$, and a high frequency part, $x_{\mathrm{H}}(\mathrm{k})$, and likewise the near-end signal, $y(k)$, is divided into $y_{\mathrm{L}}(k)$ and $y_{\mathrm{H}}(k)$. The low frequencies signals $x_{\mathrm{L}}(k)$ and $y_{\mathrm{L}}(k)$ are processed in a conventional AEC. The operation performed on the high frequency signals $x_{\mathrm{H}}(k)$ and $y_{\mathrm{H}}(k)$ will be an adaptive attenuation of $y_{\mathrm{H}}(k)$ by a gain factor, $G(k)$, resulting in a damped signal, $y_{\mathrm{G}}(k)$. The adaptation of $G(k)$ is processed by a Control Unit (CU), which sets the value of $G(k)$ depending on the value of some chosen measure of the $x_{\mathrm{H}}(k)$ signal, e.g. an estimation of the mean of $\left|x_{\mathrm{H}}(k)\right|$.

\section{Far-end side Near-end side}

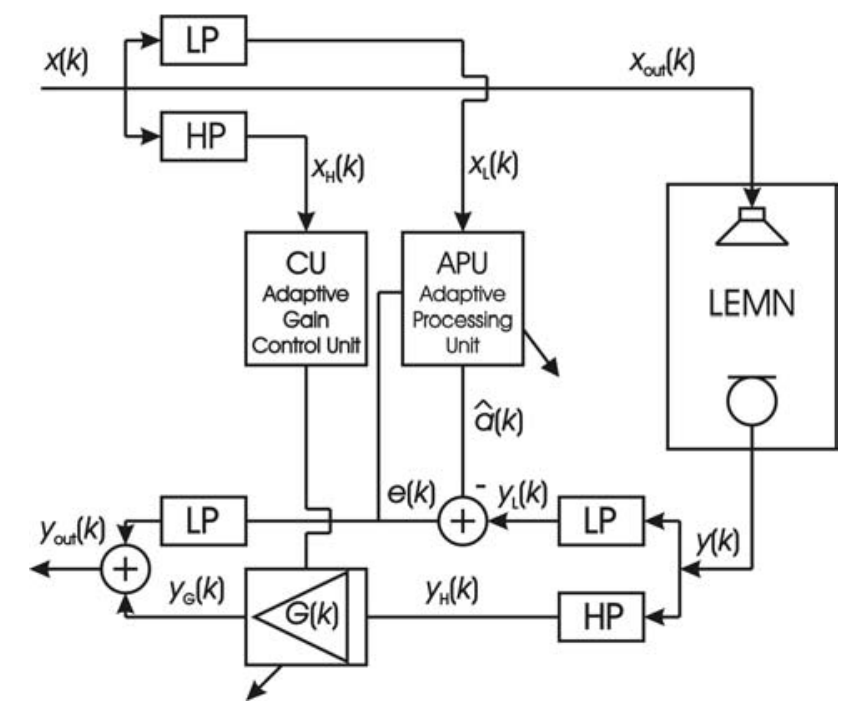

Fig. 4. The proposed solution

\section{Algorithm for the Control Unit}

In a real system there is also a delay between the arrival of a far-end signal $x_{\mathrm{H}}(k)$ and the high frequency part of acoustic echo. This delay is mainly due to the traveling time from the loudspeaker to the microphone. The gain factor $G(k)$ should adapt depending on the level of $x_{\mathrm{H}}(k)$. The adaptation needs to be rapid enough to provide a satisfying value of $G(k)$ upon the arrival of the acoustic echo. After a period of a high level $x_{\mathrm{H}}(k)$ signal, the acoustic echo can be present even if $x_{\mathrm{H}}(k)$ is zero, i.e. the loudspeaker is silent, depending on the delay in the LEM system. Thus, $G(k)$ should not be immediately increased as a response to a decrease of the $x(k)$ level. In order to meet a reasonable behavior, the following adaptation is proposed for $G(k)$,

$$
\begin{aligned}
& G(k)=\left(1-\alpha_{1}\right) G(k-1)+\alpha_{1} f(k) \text { if } f(k)>G(k-1) \\
& G(k)=\left(1-\alpha_{2}\right) G(k-1)+\alpha_{2} f(k) \text { if } f(k) \leq G(k-1)
\end{aligned}
$$

where $f(k)$ is a function of $\left|x_{\mathrm{H}}(k)\right|$, to be defined below, and $\alpha_{1}$ and $\alpha_{2}$ are two parameters determining the pace of updating $G(k)$. The parameter $\alpha_{1}$ should be set considering the distance between the microphone and the loudspeaker. A small distance enables a faster adaptation and thus a larger value of $\alpha_{1}$. If the loudspeaker and the microphone are mounted in the same shell, a fix $\alpha_{1}$ can be used, otherwise $\alpha_{1}$ should be adaptive or set to a worst-case value. The parameter $\alpha_{2}$ should be set considering the general characteristics of a typical LEM system where the phone is going to operate. The longer delay of the LEM, the lower value of $\alpha_{2}$ should be used. Typically $\alpha_{2}$ should be set considering a worst-case scenario. The function $f(k)$ should be selected considering the following aspects. In a near-end talk situation, $x_{\mathrm{H}}(k)$ only consists of low energy background noise and the acoustic echo is not significant, i.e. no damping is needed. Further, if the background noise of $x_{\mathrm{H}}(k)$ is allowed to modify $G(k)$ in situations of near-end talk, the near-end speech signal might be distorted. Thus, for values of $\left|x_{\mathrm{H}}(k)\right|$ below a certain limit, say $x_{0}, G(k)$ should be adapted towards unity. If the $x_{\mathrm{H}}(k)$ level increases, $G(k)$ should decrease. However, decreasing $G(k)$ below a certain level will not improve the quality of the system. Since there is a limit to the sound intensity from the loudspeaker there is also a limit for the value of $G(k)$ required. Further, setting $G(k)=0$, might introduce artifacts as unnatural background noise. Thus, for values of $\left|x_{\mathrm{H}}(k)\right|$ beyond a certain limit, say $x_{1}, G(k)$ should be adapted towards a lowest value, denote $G_{\text {LOW }}$. Thus $f(k)$ can be defined as

$$
f(k)=\left\{\begin{array}{lll}
1 & \text { if } & 0 \leq\left|x_{H}(k)\right|<x_{0} \\
t\left(\left|x_{H}(k)\right|\right) & \text { if } & x_{0} \leq\left|x_{H}(k)\right|<x_{1} \\
G_{\text {LOW }} & \text { if } & x_{1} \leq\left|x_{H}(k)\right|
\end{array}\right.
$$

where $t\left(\left|x_{\mathrm{H}}(k)\right|\right)$ is a transition function, i.e. a function connecting the points $\left(x_{0}, 1\right)$ and $\left(x_{1}, G_{\mathrm{LOW}}\right)$. A versatile function, $t(y) y \in[0,1]$, which connects the points $(0,1)$ and $(1,0)$ is defined by

$$
t(y)=\left[1-y^{\beta}\right]^{1 / \beta}
$$

where $\beta$ is a parameter determining the shape of the function $t(y)$. By rescaling (4) in order to fit $f(k), t(|x(k)|)$ is obtained as 


$$
t(|x(k)|)=\left[1-\left(\frac{|x(k)|-x_{0}}{x_{1}-x_{0}}\right)^{\beta}\right]^{\frac{1}{\beta}}\left(1-G_{\mathrm{LOW}}\right)+G_{\mathrm{LOW}}
$$

An example of values of $f(k)$ for different values of $\left|x_{\mathrm{H}}(k)\right|$ is given in Fig.5. The adaptation of $G(k)$ is thus defined through (1), (2), (3), and (5).

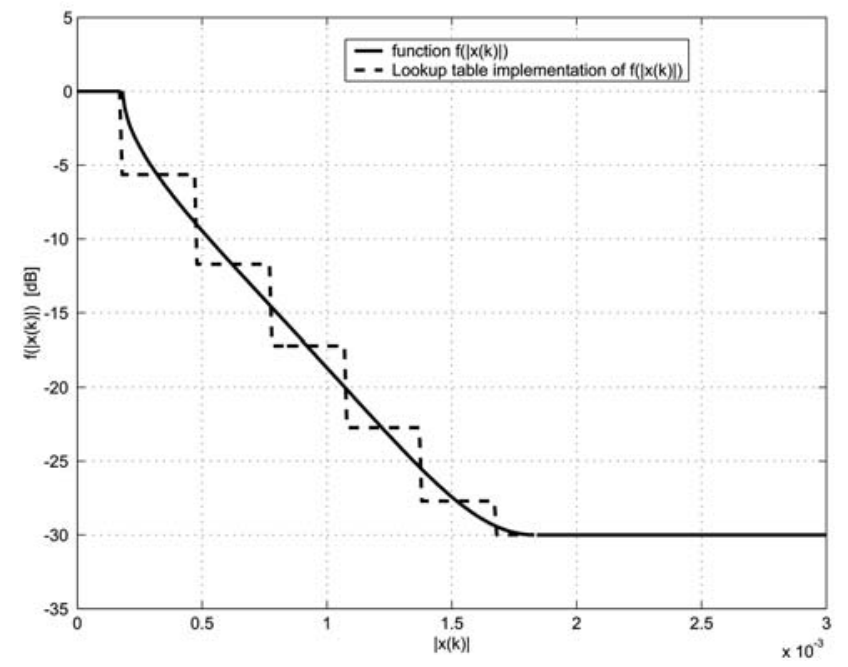

Fig. 5. The function $f(k)$ and a lookup table version thereof for the parameter values $x_{0}=0.00055, x_{1}=0.0055, \beta=0.5$, and $G_{\text {LOW }}=-30 \mathrm{~dB}$.

\section{Complexity Analysis}

An AEC solution operating with a communication bandwidth, $B$, will require a sampling frequency $f_{s}$, with $f_{s}>$ $2 B$. With an echo canceling duration of $T$ seconds, the NLMS algorithm will require an adaptive Finite Impulse Response (FIR) filter of length $N=T f_{\mathrm{s}}$. For every sample the echo canceler will require $N$ Digital Signal Processor (DSP) instructions for the filtering and $2 N$ DSP instructions for the update of the coefficients of the adaptive filter. This gives that the total number of DSP instructions per second for the AEC method, $I_{B}$, is given by

$$
I_{B}=3 T\left(f_{s}\right)^{2}
$$

If the bandwidth is to be straight-forwardly extended to $2 B$, and still use the same structure, both the sampling frequency and the filter length need to be increased by a factor 2 . The complexity, $I_{2 B}$, is thus given by

$$
I_{2 B}=3 T\left(2 f_{s}\right)^{2}
$$

i.e. the complexity is increased a factor 4 . If the proposed method is used for a bandwidth extension to $2 B$, the AEC can still operate with a sampling frequency $f_{s}$. With a communication bandwidth of $2 B$, the sampling rate of signals $x(k)$ and $y(k)$ are $2 f_{s}$. Assuming that the filtered signals $x_{\mathrm{L}}(k)$ and $y_{\mathrm{L}}(k)$ have bandwidth $B$, the AEC can then be implemented with a sampling rate of $f_{\mathrm{s}} \mathrm{Hz}$ through the use of downsampling. The processing required to implement the adaptive gain algorithm in section $B$ is insignificant since (5) can be implemented through a lookup table. Thus, the extra processing required, $I_{P}$, by the proposed method is limited to the filters required for the frequency splits/sample rate conversion. Five filters are required, see Fig.5. If FIR filters all of length $N_{P}$ are used, $I_{P}$ is given by

$$
I_{P}=5 N_{P}\left(2 f_{s}\right)
$$

Typical values for $T, f_{s}, N_{P}$ in a real industrial implementation are $T=0.250 \mathrm{~s}, f_{s}=8000 \mathrm{~Hz}$, and $N_{P}=25$ taps. Using these values in (6) - (8) gives $I_{B}=48$ Million Instructions Per Second (MIPS), $I_{2 B}=192$ MIPS, and $I_{P}=2$ MIPS. Thus comparing (6) - (8) for typical values yields that a direct expansion of the bandwidth by a factor 2 requires a factor 4 more processing capability, whereas the proposed method can extend the bandwidth with only a negligible increase in computational demand.

\section{REAL-TIME IMPLEMENTATION}

\section{A. Implementation}

In order to evaluate the proposed method two real-time systems were implemented. The first system, denoted $S$, is an implementation of an NLMS based AEC as described in IV. The second system is an extension of $S$, denoted $S_{\mathrm{EXT}}$, according to the method presented in V. The two systems were both implemented on the same fix-point AD-2186 processor, [12]. The use of a single processor allows an instant switch between the two systems, which facilitated the evaluation. The communication bandwidth of system $S$ was $[300 \mathrm{~Hz}, 3200 \mathrm{~Hz}]$ and the bandwidth of system $S_{\mathrm{EXT}}$ was $[300 \mathrm{~Hz}, 7000 \mathrm{~Hz}]$. These limits were chosen considering the standards for PSTN and ISDN, respectively, see [13], [14]. The parameter values used in the real-time implementation are given in table I.

\section{TABLE I}

PARAMETERS AND CORRESPONDING VALUES

\begin{tabular}{cc}
\hline \hline Parameter & Value \\
\hline$\alpha_{1}$ & 0.3 \\
$\alpha_{2}$ & 0.0009 \\
$x_{0}$ & 0.00055 \\
$x_{1}$ & 0.0055 \\
$\beta$ & 0.5 \\
$G_{\text {LOW }}$ & $-30[\mathrm{~dB}]$ \\
\hline
\end{tabular}

The function $t(|x(k)|)$ given in (5) was implemented using a lookup table, see Fig. 5. 


\section{B. Setup}

An omnidirectional microphone was used to obtain the nearend input signal. Three loudspeakers were used to produce the near-end output signal. The loudspeakers were placed in a circle surrounding the microphone in order to resemble a conventional omnidirectional conference phone, see Fig.6. The far-end input signal was fed to a headset, placed in an other room, in order to provide acoustic isolation. The far-end output signal was obtained by a hand-held microphone and delayed $200 \mathrm{~ms}$ by a delay circuit. The delay was done in order to simulate the delay in telephone wires and switching offices, and to make acoustic echoes clearly audible at the far-end side. The setup was done in a small office with a reverberation time of approximately $500 \mathrm{~ms}$ expressed with RT60, where RT60 defines the reverberation time it takes for the sound level in a room to decrease with $60 \mathrm{~dB}$ after an impulse.

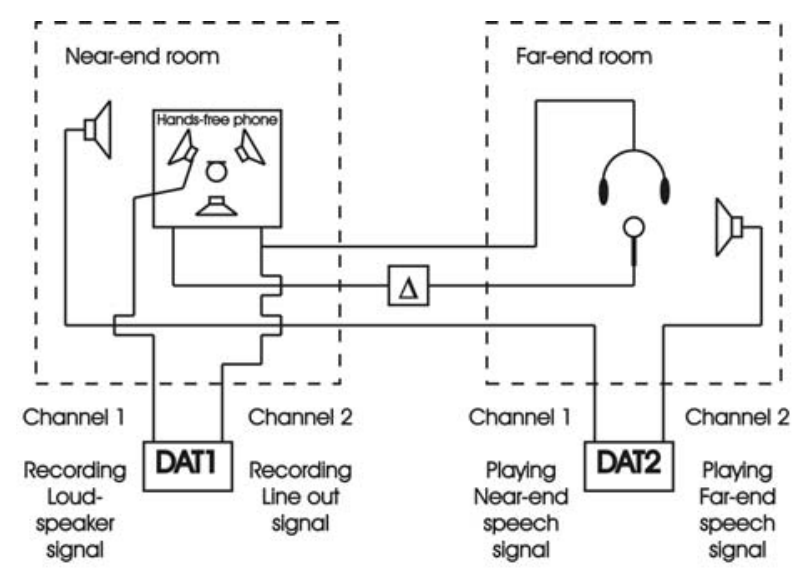

Fig. 6. The measurement setup

\section{Evaluation}

In order to obtain a set of near-end and far-end speech signals with corresponding phone loudspeaker and phone lineout signals, two DAT recorders, DAT1 and DAT2, were used, see Fig.6. Channel 1 of DAT1 was used to record the loudspeaker signal, channel 2 of DAT1 was used to record the phone line out signal, channel 1 of DAT2 was used to play the near-end speech signal, and channel 2 of DAT2 was used to play the far-end speech signal. The phone was set to solution $S$ mode, a tape was played on DAT2, and at the same time DAT1 was recording. The DAT2 tape contained a session of: idle, near-end talk, far-end talk, and doubletalk, see Fig.6. The procedure was repeated with the phone set in solution $S_{\mathrm{EXT}}$ mode.

A subjective real-time evaluation of the both methods was also performed. The subjective evaluation was performed in the following manner. One person placed her/himself at the near-end side, and another person placed her/himself at the far-end side. These persons performed a natural conversation, containing sessions of doubletalk. Throughout the test repeated switches between solution $S$ and solution $S_{\mathrm{EXT}}$ mode were performed.

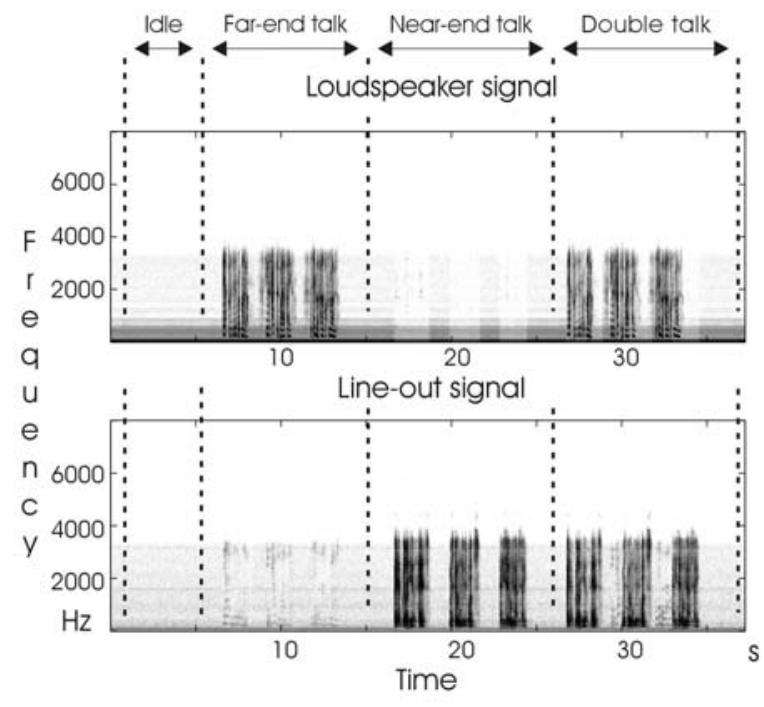

Fig. 7. Spectrograms of the conventional AEC solution

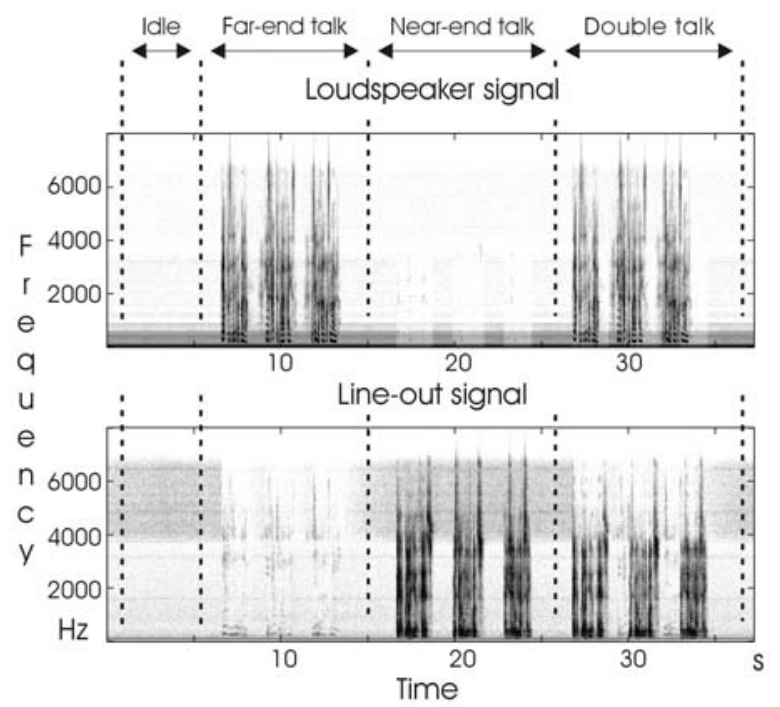

Fig. 8. Spectrograms of the proposed solution

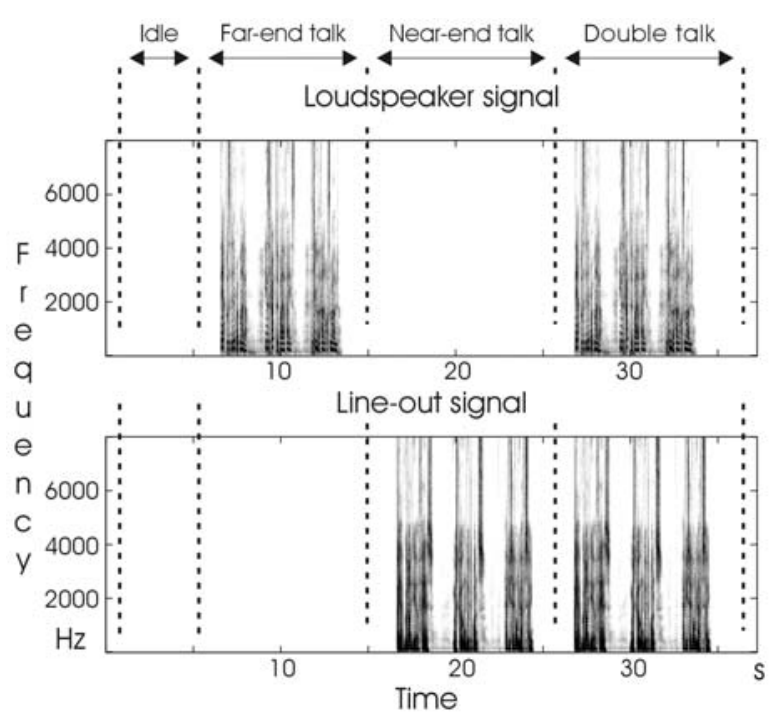

Fig. 9. Spectrograms of an ideal solution 


\section{Result}

Spectrograms of the loudspeaker and line-out signals for the conventional solution $S$ are presented in Fig.7 and for the proposed solution $S_{\mathrm{EXT}}$ in Fig.8. Note that in the phone lineout signal of the proposed solution, the upper band background noise is of a higher level, as compared to that of the lower band. This difference is due to a center-clipper noise reduction that was implemented only in the low frequency band. The spectrograms of the near-end and far-end input speech signals are shown in Fig.9, i.e. in Fig.9 the ideal, perfect frequency characteristics for the two solutions are presented. Comparing the spectrograms in Fig.7-Fig.9 shows that the proposed method gives a more natural sounding signal in that it also contains high frequency signals. The subjective tests of the two systems using real-time two-way communication showed that the extended bandwidth of the proposed system significantly increases the perceived quality. Further, the subjective tests showed that no artifacts as e.g. half-duplex characteristics are introduced by the proposed method. In [15] .wav files of the signals presented in Fig.7-9 can be found.

\section{CONCLUSION}

A method for the extension of the bandwidth of a conventional AEC conference phone was proposed and tested in real-time. The core of the method is to split the processed signals in two frequency bands, where an AEC unit processes the low frequencies and an adaptive gain unit processes the high frequencies. The proposed method has been examined for bandwidth extension from an upper limit of $3.2 \mathrm{kHz}$ to 7 $\mathrm{kHz}$. Subjective tests showed that the proposed method did not introduce artifacts, as e.g. perceived half-duplex features. The extra computational load was insignificant. Thus, the proposed method was shown to be a cost effective way of increasing the quality of a conference phone. An interesting topic for further studies is to examine if the proposed method can be used for reduction of the computational load of an AEC conference phone, i.e. using the method to reduce the bandwidth of the AEC and thereby reduce the computational complexity.

\section{ACKNOWLEDGMENT}

The authors thank the Swedish Knowledge Foundation for support throughout the research project that lead to this paper. The authors also thank the staff at Konftel Technology AB for their contributions in form of evaluation of the proposed system.

\section{REFERENCES}

[1] S. Haykin, Adaptive Filter Theory, 4th ed., Prentice-Hall, 2002.

[2] B. Widrow, S. D. Stearns, Adaptive signal processing, Prentice-Hall 1985.

[3] S. L. Gay, S. Tavathia, "The fast affine projection algorithm", Proc. IEEE ICASSP'95, vol. 5, Detroit, MI, pp. 3023-3026, May 1995.
[4] D. T. M. Slock, T. Kailath, "Numerically stable fast transversal filters for recursive least squares adaptive filtering", IEEE Transactions on Signal Processing, vol. 39, no. 1, pp. 92-114, 1991.

[5] J. M. Coiffi, T. Kailath, "Fast recursive least squares transversal filters for adaptive filtering", IEEE Transactions on Acoustic, Speech, and Signal Processing, vol. ASSP-32, no. 2, pp. 304-337, 1984.

[6] A. Mader, H. Puder, G. U. Schmidt, "Step-size control for acoustic cancellation filters - an overview", Signal Processing, vol. 80, pp. 16971719,2000

[7] P. Heitkamper, M. Walker, "Adaptive gain control for speech quality improvement and echo suppression", IEEE ISCAS'93, pp. 455-458, 1993.

[8] Ericsson: Integrated circuits data book, IC2, 1989/90.

[9] Siemens: Semiconductors for wired telecom systems, IC03b, 1998.

[10] Motorola: Semiconductors, DLE136R1/D, 1988.

[11] S. Furui, Digital speech processing, synthesis and recognition, Marcel Dekker, Inc, 1989.

[12] Analog Devices: ADSP-2100 Family user's manual, 1995.

[13] "TBR21", European Telecommunications Standards Institute, 1998.

[14] "G.722", ITU-T Recommendation ITU-T G.722.1, 1999

[15] http://www.bth.se/fou

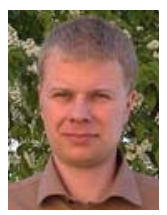

Fredric Lindstrom (S'02) was born in Skelleftea, Sweden. He received his M.S. degree in applied physics from Uppsala University, Uppsala, Sweden, in 2001. Since 2000 he has been with Konftel Technology AB, Department of Research and Development. His current research interest is adaptive signal processing with applications in hands-free systems, e.g. acoustic echo canceling, adaptive gain techniques, and algorithms for finite precision implementations.

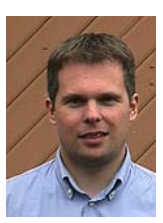

Mattias Dahl (S'94-A'95) was born in Uddevalla, Sweden. He received the B.S. degree in electrical engineering from Chalmers Institute of Technology, Gotenburg, Sweden, the M.S. degree in telecommunication and signal processing from Lulea University of Technology, Lulea, Sweden, and the Ph.D. in signal processing from Blekinge Institute of Technology, Ronneby, Sweden, in 1988, 1993, and 2000, respectively. Since 1993, he has been with the Department of Signal Processing, Blekinge Institute of Technology, Ronneby, Sweden, where he is involved in adaptive beamforming, speech enhancement, and active noise control research projects.

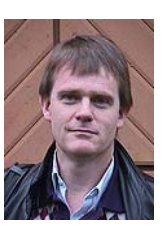

Ingvar Claesson (M'91) was born in Broby, Sweden, in 1957. He received the Dipl. Eng. and Ph. D. degrees from Lund University, Lund, Sweden, in 1980 and 1986, respectively. He was appointed Senior Lecturer in Telecommunication theory at Lund University in 1986, and was made associate Professor in 1992. Since May 1998, he has held the Chair of Signal Processing at Blekinge Institute of Technology, Ronneby, Sweden. In 1990 he was one of the founders of the Department of Signal Processing Blekinge Institute of Technology, and is currently Head of Research and Principal Supervisor in Signal Processing there. His current research interests are in adaptive signal processing, blind equalization, adaptive beamforming, speech enhancement, active noise control, filter design, and antenna arrays. 